\title{
Obtaining reliable source locations with time reverse imaging: limits to array design, velocity models and signal-to-noise ratios
}

\author{
Claudia Werner ${ }^{1,2}$ and Erik H. Saenger ${ }^{1,2}$ \\ ${ }^{1}$ International Geothermal Center Bochum, Lennershofstr. 140, 44801 Bochum, Germany \\ ${ }^{2}$ Ruhr-University Bochum, Universitätsstr. 150, 44801 Bochum, Germany \\ Correspondence: Claudia Werner (claudia.werner@hs-bochum.de)
}

Received: 27 July 2018 - Discussion started: 21 August 2018

Revised: 3 November 2018 - Accepted: 1 December 2018 - Published: 14 December 2018

\begin{abstract}
Time reverse imaging (TRI) is evolving into a standard technique for locating and characterising seismic events. In recent years, TRI has been employed for a wide range of applications from the lab scale, to the field scale and up to the global scale. No identification of events or their onset times is necessary when locating events with TRI; therefore, it is especially suited for locating quasi-simultaneous events and events with a low signal-to-noise ratio. However, in contrast to more regularly applied localisation methods, the prerequisites for applying TRI are not sufficiently known.

To investigate the significance of station distributions, complex velocity models and signal-to-noise ratios with respect to location accuracy, numerous simulations were performed using a finite difference code to propagate elastic waves through three-dimensional models. Synthetic seismograms were reversed in time and reinserted into the model. The time-reversed wave field back propagates through the model and, in theory, focuses at the source location. This focusing was visualised using imaging conditions. Additionally, artificial focusing spots were removed using an illumination map specific to the set-up. Successful locations were sorted into four categories depending on their reliability. Consequently, individual simulation set-ups could be evaluated by their ability to produce reliable source locations.

Optimal inter-station distances, minimum apertures, relations between the array and source locations, heterogeneities of inter-station distances and the total number of stations were investigated for different source depths and source types. Additionally, the accuracy of the locations was analysed when using a complex velocity model or a low signalto-noise ratio.
\end{abstract}

Finally, an array in southern California was investigated regarding its ability to locate seismic events at specific target depths while using the actual velocity model for that region. In addition, the success rate with recorded data was estimated.

Knowledge about the prerequisites for using TRI enables the estimation of success rates for a given problem. Furthermore, it reduces the time needed to adjust stations to achieve more reliable locations and provides a foundation for designing arrays for applying TRI.

\section{Introduction}

The localisation and characterisation of seismic events in the subsurface are crucial for understanding physical processes in the Earth. Well-established methods are able to locate most seismic events in a fast and reliable manner; however, they rely on the identifiable onsets of events. Time reverse imaging (TRI) is a method especially suited for locating and characterising events which are indistinguishable in traces because they occur quasi-simultaneously or are superposed by noise. The prerequisites for more regularly applied localisation methods are very well known. However, the station distributions, the degree of complexity in the velocity model and the level of noise that hinder or enhance locating events with TRI are not sufficiently known. Therefore, this study systematically tests different station distributions for their localisation capabilities while considering complex velocity models and low signal-to-noise ratios.

TRI uses the whole recorded waveform rendering the identification of events and their onsets obsolete. It can be applied 
as long as the wave propagation can be described with a timeinvariant wave equation. Seismic traces are reversed in time and back propagated through a medium until they focus on the initial event location. Imaging conditions are used to visualise aspects of the back-propagating wave field and obtain the source location.

TRI has been applied in earth sciences as well as medical sciences for some time (Fink et al., 1999). In recent years multiple studies have shown that TRI is a reliable, easyto-use localisation tool: it has been used to retrieve source information on various scales from the lab scale in nondestructive testing (Saenger, 2011; Anderson et al., 2011; Harker and Anderson, 2013; Kocur et al., 2016), over the field scale, for example in volcanic tremor (Lokmer et al., 2009) and non-volcanic tremor applications (Larmat et al., 2009; Horstmann et al., 2015) and above hydrocarbon reservoirs (Steiner et al., 2008), up to the global scale (Larmat et al., 2006, 2008). However, to apply TRI, a fairly accurate velocity model is needed to precisely back propagate the wave field. With the increasing availability of high resolution large three-dimensional velocity models and growing knowledge about prerequisites, TRI has the potential to locate seismic events, which could previously not be reliably located, over a wide range of applications.

\subsection{Restrictions to the localisation capabilities of TRI}

The estimation of location accuracy is one major challenge when applying TRI. Therefore, a common approach is to perform a preliminary synthetic study to test if the given velocity model and station distribution enable reliable locations. If the synthetic study fails, the set-up is adjusted until either the study is abandoned or a sufficiently reliable result is achieved. This adjustment phase can be time-consuming because there are multiple characteristics appearing at once that may hinder the localisation.

Numerous characteristics regarding where stations are placed at the surface seem to significantly influence the chance of success with TRI. In theory, TRI works with only one station (Montagner et al., 2012), although in most cases more stations are needed to obtain a stable result. Therefore, the total number of stations is often considered to have a major influence on the success or failure of obtaining accurate source locations (Kremers et al., 2011; Horstmann et al., 2015). However, numerous reports state that a smaller number of stations works just as well (Gajewski and Tessmer, 2005; Steiner et al., 2008; Lokmer et al., 2009; Larmat et al., 2009).

The aperture of the array seems to be another characteristic of the station distribution that influences location accuracy. Steiner et al. (2008) and Lokmer et al. (2009) have reported false locations in a two-dimensional case when the aperture of the array is limited. Moreover, Artman et al. (2010) emphasised the importance of a sufficiently large aperture to obtain a spatially focused location.
Distances between the individual stations and the homogeneity of these inter-station distances seem to also be important (Lokmer et al., 2009). Furthermore, the azimuthal gap, which is the angle between two stations viewed from the epicentre location, is introduced by Horstmann et al. (2015). It is an indirect measurement of the heterogeneity of inter-station distances. Horstmann et al. (2015) observed an enhanced localisation result if the maximum azimuthal gap was small. Lokmer et al. (2009) and Horstmann et al. (2015) also noted a different quality of focusing for sources at different depths while using the same stations.

In addition to the placement of stations, the velocity model can significantly influence the success rate of TRI. A velocity model that differs from the true velocity model may inhibit the localisation of real events. However, if the error is small enough, TRI is still successful (Gajewski and Tessmer, 2005; Lokmer et al., 2009; Saenger, 2011). Similarly, a smoothed velocity model does not significantly influence the results (Gajewski and Tessmer, 2005). Furthermore, complex velocity structures such as low-velocity zones or sharp velocity contrasts may inhibit the application of TRI even when using synthetic data (Larmat et al., 2009). Nevertheless, necessary simplifications due to the availability of velocity models may inhibit the localisation of real events using TRI (Artman et al., 2010; Horstmann et al., 2015). Common simplifications include the utilisation of a constant ratio of P-wave velocity to $S$-wave velocity or a constant density.

A high level of noise in the traces may inhibit localisation using TRI, even when the array and the velocity model are sufficient. In theory, TRI even works with a very low signalto-noise ratio, as noise is random and will not superpose constructively to form a focus during the back propagation of the wave field. Gajewski and Tessmer (2005) showed traces with events that were indistinguishable from the noise, and TRI was still able to reconstruct the source location. Witten and Artman (2011) created synthetic data with signal-to-noise ratios as low as 0.25 and showed successful localisations. Nevertheless, they observed a decrease in location accuracy with lower signal-to-noise ratios.

\subsection{Objective of this study}

This study aims to find station distributions that produce reliable source locations with TRI. Finding these optimal station distributions is crucial to estimate the success rate of TRI with a given set of stations. In addition, the time needed to adjust station distributions may be decreased.

Furthermore, the prerequisites of the method should be known when designing an array for TRI. Therefore, the influence of the station distribution, the complexity of the velocity model and the effect of different levels of noise on the location accuracy were investigated.

We performed numerous simulations to systematically analyse different station distributions and their influence on the location accuracy of sources at different depths as well 
as analysing the influence of different source types. Therefore, the focus was on the distance between receivers, the symmetry of the array in relation to the source position, the azimuthal gap between receivers and the number of stations. Simulations were first performed with a homogeneous velocity model and then with a complex velocity model. Location accuracy was investigated when the velocity model was known and when it was not specifically known. We investigated the ability of TRI to cope with very low signal-tonoise ratios. To complete the study, we applied the guidelines found using the methodical tests to an actual real-life example in southern California. The ability to locate events at a target depth was investigated while using the existing array as well as the actual velocity model for the specific region from Zeng et al. (2016).

Using the southern Californian example, we demonstrate how to assess the success rate of the localisation with TRI using a synthetic study. Additionally, the station distribution was altered to enhance location accuracy. We give estimates regarding the part of the model in which reliable locations can be expected. Additionally, we demonstrate how to design an array that produces reliable source locations.

All simulations were performed using a finite difference scheme to propagate elastic waves through threedimensional models. The advantage of a purely synthetic study is that the source location is known, meaning that the location accuracy can then subsequently be estimated. Additionally, parameters influencing the localisation can be tested individually.

\section{The method of time reverse imaging (TRI)}

Time reverse imaging (TRI) uses the whole waveform of recorded seismograms to locate and characterise seismic events. The method consists of three steps following the workflow introduced by Saenger (2011) and modified by Witten and Artman (2011): the reversal of individual traces in time, the back propagation of the time-reversed wave field and the elimination of artefacts impinged by the velocity structure and model set-up. Results are then visualised using suitable imaging conditions. In the following, the adaptation of this workflow to this study is described in detail.

The TRI method was investigated using synthetic seismograms created by forward simulations of the propagation of elastic waves through a suitable medium. In this study, a homogeneous model was first used for the synthetic tests and the southern California example which was then followed by a heterogeneous velocity model for the same region. The seismograms obtained were not altered besides flipping them in time. Montagner et al. (2012) used binarised seismograms to demonstrate that TRI is based on the coherency of the phases in the seismograms and not on the amplitudes. Therefore, the phase information in the recorded seismograms should be kept as close to the original as possi- ble, and any filter used should be a zero-phase filter to prevent phase shifts in the time domain. Additionally, all traces need to be time-synchronous.

The time-reversed seismograms were reinserted into the model domain at the exact locations where they were recorded. The receivers of the forward simulation act as sources in the time-reversed simulation. In this study, receivers always mean stations at the surface which act as receivers in forward simulations and sources in reverse simulations. The time-reversed wave field back propagates through the model and collapses at the initial source location.

Imaging conditions highlight specific characteristics of the back-propagating wave field. They were used to visualise the point in space and time where the wave field focused. In this numerical study, the event time was known and the positions of foci were compared to the source position used in the forward simulation to determine the accuracy of the event locations.

Apart from the focusing spot at the initial source location, parts of the velocity structure and the model domain itself may be highlighted by artificial focusing spots. Usually, artificial focusing spots appear around the receiver positions at the surface of the model. Additionally, special velocity structures, such as low velocity zones, may cause artificial focusing (Larmat et al., 2009). To eliminate those artefacts, the workflow introduced by Witten and Artman (2011) was used. Images produced with the specific imaging conditions were divided by images produced by the back propagation of random noise. To keep amplitudes and frequency content the same, recorded seismograms were back propagated through the model not time-reversed. The non-time-reversed wave field cannot focus on the initial source location and acts as a noise field. The result is an illumination map of the model highlighting areas where focusing occurs solely due to the velocity structure and the model set-up used as described by Witten and Artman (2011). When dividing the results by this illumination map ideally only focusing spots created by interference from the time-reversed traces remain in the image.

\subsection{Simulation of wave propagation in elastic media}

The wave propagation in three-dimensional elastic media for the forward and the backward simulations was performed using a finite difference code developed by Saenger et al. (2000). A second-order finite-difference operator was used in space and in time. The explicit finite difference operators used for the rotated staggered grid are discussed in Saenger et al. (2000). The model boundaries at the bottom and at the sides of the models were absorbing boundaries following Clayton and Engquist (1980), and the top boundary was a free surface that is incorporated as a vacuum layer.

A non-volcanic tremor application in southern California was used as a real-life example. Therefore, the models used in this study were set up to be numerically stable when utilising the receivers used in Horstmann et al. (2015) and the 
updated velocity model by Zeng et al. (2016) which covers the same region used in Horstmann et al. (2015). All models span from -65 to $+65 \mathrm{~km}$ in the $x$ direction, from -70 to $+50 \mathrm{~km}$ in the $y$ direction and from the surface at $0 \mathrm{~km}$ to a depth of $28 \mathrm{~km}$. The grid spacing was set to $0.1 \mathrm{~km}$ to balance accuracy and stability in the simulations, while the time step was set to $0.01 \mathrm{~s}$. For the synthetic tests, a homogeneous velocity model with the same dimensions as the velocity model from southern California was created.

Sources in the forward simulations were implemented as moment tensor sources. An explosion source (only $M_{x x}=$ $M_{y y}=M_{z z}$ were non-zero and all were equal in strength) or a strike-slip source (only $M_{x y}=M_{y x}$ was non-zero) was used. As a source signal, the negative normalized second derivative of the Gaussian function, also called a Ricker wavelet, was used. The Ricker wavelet was implemented following Shearer (2009):

$R(t)=\left(1-2 \pi^{2} f_{\mathrm{p}}^{2} t^{2}\right) \cdot \exp \left(-\pi^{2} f_{\mathrm{p}}^{2} t^{2}\right)$,

where $t$ denotes the time and $f_{\mathrm{p}}$ the peak frequency of the wavelet. For all simulations in this study the peak frequency was set to $f_{\mathrm{p}}=1.75 \mathrm{~Hz}$ which resulted in a maximum frequency of about $f_{\max }=5 \mathrm{~Hz}$. Non-volcanic tremors are usually observed in this frequency range (Horstmann et al., 2015). The wavelet was centred at $t=0 \mathrm{~s}$.

The source position was set to $x=-1.8 \mathrm{~km}$ and $y=$ $-28.3 \mathrm{~km}$, which corresponds to an existing recorded earthquake that was randomly chosen and represents one possible source location. The earthquake occurred on the 28 June 2011 at 14:10 and had a magnitude of 1.68 (NCEDC, 2014). The three depths used for the sources were: $z_{1}=$ $5 \mathrm{~km}, z_{2}=11.9 \mathrm{~km}$ and $z_{3}=22 \mathrm{~km}$. The deepest source represents the depth where non-volcanic tremors occur, the medium depth is the depth of the example earthquake, and the shallow source represents a shallow end-member that is not geologically relevant in the chosen region in southern California.

\subsection{Imaging conditions}

Imaging conditions were used to visualise specific characteristics of the back-propagated wave field. They were calculated as the maximum value of specific characteristics of the wave field at each point $\boldsymbol{x}$ of the model domain over the whole simulation time, from $t=0 \mathrm{~s}$ to $t=T$. A perfect result would be a single grid point with the highest value of the specific imaging condition at the initial source location and very low values everywhere else.

Numerous imaging conditions have been proposed in the past. Here we summarise characteristics of the wave field that can be used to calculate imaging conditions. The most intuitive imaging condition uses the maximum amplitude of displacement (e.g. used in Gajewski and Tessmer, 2005; Larmat et al., 2009; and Saenger, 2011). The maximum particle velocity is also a suitable imaging condition (Steiner et al.,
2008; Lokmer et al., 2009). Alternatively, the divergence and the curl field can be used for imaging conditions that are sensitive to P-waves and S-waves, respectively (Larmat et al., 2009; Lokmer et al., 2009; Horstmann et al., 2015). The strain field has the potential to directly reveal source characteristics (Larmat et al., 2009; Kremers et al., 2011). Additionally, energy densities can be computed from the parameters mentioned above (Artman et al., 2010; Saenger, 2011).

In this study, we focused on the four imaging conditions proposed by Saenger (2011). The maximum particle displacement $\mathbf{I}_{\mathbf{d}}$ was calculated using

$\mathbf{I}_{\mathrm{d}}(\boldsymbol{x})=\max _{t \in[0, T]}\|\boldsymbol{u}(\boldsymbol{x}, t)\|$,

with $\boldsymbol{u}(\boldsymbol{x}, t)$ being the displacement at each point $\boldsymbol{x}$ in the model at each time $t$.

The maximum $\mathrm{P}$ - and S-wave energy density imaging conditions $\left(\mathbf{I}_{\mathrm{p}}\right.$ and $\mathbf{I}_{\mathrm{s}}$ ) were calculated by separating the wave field into a divergence field and a curl field, followed by the calculation of the energy densities after Dougherty and Stephen (1988):

$$
\begin{aligned}
& \mathbf{I}_{\mathrm{p}}(\boldsymbol{x})=\max _{t \in[0, T]}(\lambda+2 \mu)[\nabla \cdot \boldsymbol{u}(\boldsymbol{x}, t)]^{2}, \\
& \mathbf{I}_{\mathrm{S}}(\boldsymbol{x})=\max _{t \in[0, T]} \mu[\nabla \times \boldsymbol{u}(\boldsymbol{x}, t)]^{2} .
\end{aligned}
$$

$\lambda$ and $\mu$ represent the Lamé parameters. The maximum total energy density imaging condition $\mathbf{I}_{\mathrm{e}}$ was based on the multiplication of stresses and strains at every point in the model domain:

$$
\mathbf{I}_{\mathrm{e}}(\boldsymbol{x})=\max _{t \in[0, T]} \sum_{i} \sum_{j}\left[\sigma_{i j}(\boldsymbol{x}, t) \varepsilon_{i j}(\boldsymbol{x}, t)\right] .
$$

$\sigma_{i j}(\boldsymbol{x}, t)$ are the stresses and $\varepsilon_{i j}(\boldsymbol{x}, t)$ are the strains at each point $\boldsymbol{x}$ in the model domain at each time $t$.

Each of these four imaging conditions were calculated with a different characteristic of the wave field. Therefore, they enable the independent inspection of the focus point.

\subsection{Evaluation of location success}

In this study, we aim to find prerequisites for the application of TRI. Therefore, it is necessary to be able to evaluate the reliability of the focusing. TRI claims to work with no a priori information regarding the number of events or their positions in time and space, which implies that the results from this method should enable the unambiguous localisation of events. The origin time was known for all simulations and we only used one source per simulation. Therefore, the focus was on the location accuracy.

The location of an event can be found by looking for the point with the maximum value in each imaging condition. Because the wave field was only inserted at discrete points at the surface during the back propagation, the wavefront needs to heal before being able to focus on the source location. The depth at which the wavefront is healed depends 
on the discretization of the emitted wave field (Witten and Artman, 2011). These artefacts were close to the surface and had higher amplitudes than the localisation. We observed that on average at a depth of one P-wave wavelength sampling artefacts stopped interfering with the localisation of events. Therefore, all values above the depth of one P-wave wavelength were set to zero. With the frequency range and velocity models used, this boundary depth was $2.3 \mathrm{~km}$ beneath the free surface. However, muting the upper $2.3 \mathrm{~km}$ of the model inhibits localisation of shallow sources. Depending on the source depths expected, this should be considered. In our example application in southern California, local earthquakes are not reported at depths shallower than $10 \mathrm{~km}$, while non-volcanic tremors are expected at even greater depths (Horstmann et al., 2015). Therefore, muting the shallow part of the model does not interfere with the event locations in this specific case.

To evaluate if a certain station distribution enhances reliable localisations, a two-step approach was performed: first the location accuracy was determined and locations were sorted as being close to or far away from the source; second, the intensity of artificial focusing spots was evaluated by introducing four categories. It is crucial to evaluate the intensity of artificial focusing produced with certain set-ups as they can obscure source locations. In the following, these two steps are described in more detail.

To determine if the location accuracy was adequate, a maximum average deviation threshold was introduced. Locations deviating further from the source than this threshold were considered to be unsuccessful. The average deviation was calculated as the mean of the error of the source location in all three directions. Therefore, locations could have an error in one direction up to three times the defined maximum average deviation if the other two directions had exactly the value for the original source location. Consequently, the allowed source area is a octahedron centred at the source location. The maximum average deviation threshold was empirically found to be $1.2 \mathrm{~km}$. The maximum values found in the imaging conditions were either inside the octahedron formed by this threshold or far away from it. Allowing this rather large location error enabled this study to focus on finding optimal station distributions rather than focusing on very accurate source locations. Finding an appropriate set-up to achieve the most accurate source locations possible strongly depends on the specific application and is therefore not the focus of this study.

As a second step, each imaging condition that was labelled successful in the first step was normalised by itself and then visualised. Normalising the imaging conditions allowed the direct comparison of different station distributions and different imaging conditions. When visualising the normalised imaging conditions, it proved useful to create separate plots containing all points with a certain fraction of the maximum value. Plots were created for values in incremental steps of 0.1 , corresponding to a tenth of the maximum value. By viewing all points of the imaging conditions with a specific fraction of the maximum value, successful locations could be assessed further. An example of this plot type is shown in Fig. 1. The top two plots show imaging conditions for the total energy density imaging condition $\mathbf{I}_{\mathrm{e}}$ for two different values $(0.6$ and 0.9$)$. The middle two plots show the same for the P-wave energy density imaging condition $\mathbf{I}_{\mathrm{p}}$ and the bottom two plots show the same for the maximum displacement imaging condition $\mathbf{I}_{\mathrm{d}}$.

Figure 2 is a one-dimensional representation outlining relevant aspects of the four categories. This representation is equivalent to viewing a one-dimensional profile through the location and noting the values of the imaging conditions along this profile. A peak in Fig. 2 refers to a visible focus in the image and the height of the peak represents the value at the focusing point. The top dashed line in Fig. 2 shows amplitudes of 0.9. This corresponds to Fig. 1b. All visible spots in Fig. $1 \mathrm{~b}$ translate to peaks higher than 0.9 in Fig. 2. Similarly, the bottom dashed line represents values of 0.6 or $60 \%$ (Fig. 1a). Depending on the maximum value of peaks outside of the designated source area, each imaging condition was sorted into one of four categories:

Category I included the most reliable locations. There was one peak inside the source area corresponding to the location of the event. All other peaks were below 0.6. The plots showing $\mathbf{I}_{\mathrm{e}}$ in Fig. 1 would be categorised into category I.

Category II was similar to category I but one (or more) secondary peaks were between 0.6 and 0.9 . Simulations sorted into this category were also considered to be reliable. The plots showing $\mathbf{I}_{\mathrm{p}}$ in Fig. 1 would be sorted into category II because at 0.6 there are multiple black spots and at 0.9 there was only one focus visible.

Category III is depicted in Fig. 2 with two peaks above 0.9. Both of these peaks are inside the source area. In the cases we associated with category III, there was more than one peak, but all peaks above 0.6 were within the source area. Therefore, we assumed that they belong to the same location. Depending on the type of application, station distributions producing category III locations should be considered carefully.

Category IV simulations were regarded as unsuccessful, although the highest value was found to deviate less than the threshold value from the source. Focusing spots outside of the source area had a peak value of more than 0.9. The plots for $\mathbf{I}_{d}$ in Fig. 1 would be sorted into this category. In category IV simulation set-ups, locations could not be distinguished from artificial focusing spots without knowing the initial source location.

Finally, to determine which simulation set-ups would produce reliable locations, the simulations with locations in category I and II should be viewed. Simulations with more than one imaging condition in category I or II were considered to be simulations producing reliable locations. This requirement to have two successful imaging conditions per simulation inhibits the misinterpretation of an artefact for a location. This criterion could be crucial when using real data. 

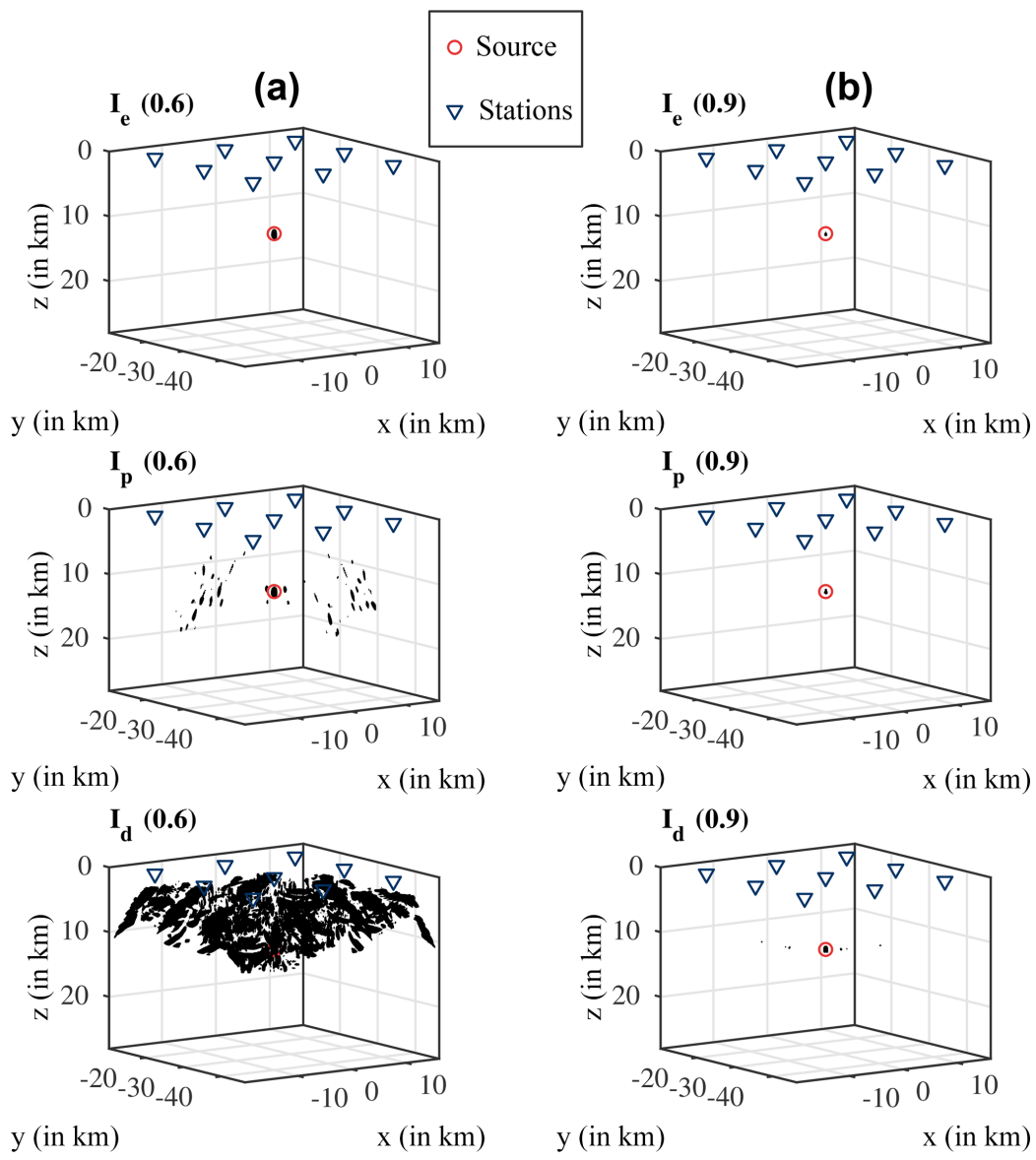

Figure 1. Imaging conditions plotted for one example simulation. Black spots are points with the values of 0.6 (in column a) and 0.9 (in column b) in the respective imaging conditions. $\mathbf{I}_{\mathrm{e}}$ would be classified into category $\mathrm{I}, \mathbf{I}_{\mathrm{p}}$ would be classified into category II and $\mathbf{I}_{\mathrm{d}}$ would be classified as category IV, which would be regarded as unsuccessful. This example shows an explosion source at a depth of $11.9 \mathrm{~km}$ with nine stations placed in a square. The inter-station distance is $13 \mathrm{~km}$. Only the part of the model underneath the stations is shown.

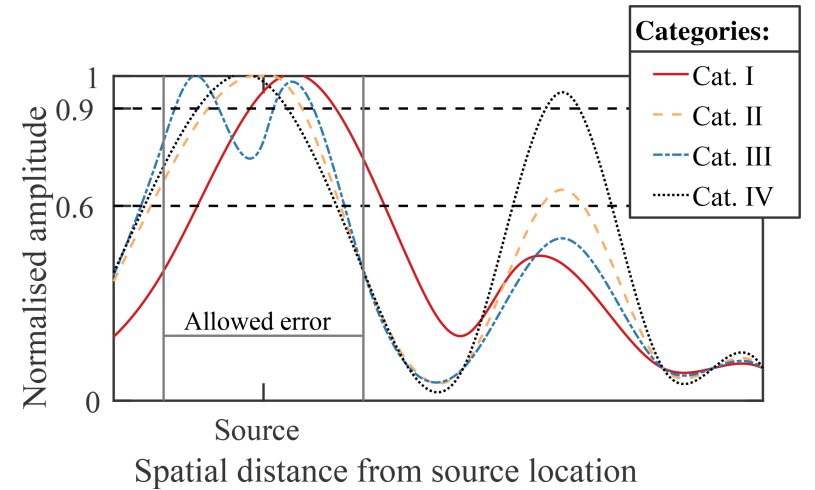

Figure 2. One-dimensional schematic outlining the four different categories that were used to rank simulation set-ups by their location accuracy.

\section{The influence of the station distribution}

To discover prerequisites for locating seismic sources using TRI, simulations were performed with varying station distributions. Whether the specific set-up enhances the localisation can be derived from the reliability of locations achieved with that set-up.

The reliability of locations is influenced by the number and position of stations at the surface. The wave field is only sampled at these discrete positions. Therefore, different aspects of the station distribution were tested systematically to determine which station placements enable a reliable and unambiguous localisation.

In the first set of simulations, optimal apertures and interstation distances were sought that still produce reliable locations. The ability to locate sources with an array not centred above the source was tested in a second set of simulations. In the third set of simulations, the ability of TRI to cope with heterogeneous station distributions was tested. 
These three sets of simulations were performed with nine receivers at the top of the model. Additionally, the effect of using an increased number of receivers was investigated for individual set-ups. All simulations in this section were performed using a homogeneous velocity model for forward as well as for time-reversed simulations. The P-wave velocity was set to $4000 \mathrm{~m} \mathrm{~s}^{-1}$, the S-wave velocity was set to $2300 \mathrm{~m} \mathrm{~s}^{-1}$ and the density was set to $2000 \mathrm{~kg} \mathrm{~m}^{-3}$. There was no noise added to the traces. Therefore, the only change between simulations was the station distribution. Hence, observed differences in location accuracy were presumably caused by the change in the station placement.

\subsection{Optimum aperture and inter-station distance}

To find the optimal apertures and maximum inter-station distances that still enabled localisation with TRI, nine stations were placed in a square and centred above the source location. The distance $(d)$ between the stations was then increased discretely (see Fig. 3). By increasing the inter-station distance, the aperture of the array was also increased. When using nine stations in a square layout, the aperture is twice the distance $(d)$ between receivers. Therefore, the expected result would be a range of receiver distances producing reliable source locations. The lower bound of this range corresponds to the minimum aperture and the upper bound to the maximum inter-station distance needed to locate a source of the specific type at the specific depth.

In Fig. 4 the simulations performed are marked with grey bars to distinguish gaps in simulations from simulations that did not locate the source successfully. Only successful locations, which deviate less than the threshold from the source location, are marked with a symbol corresponding to the imaging condition and a colour corresponding to the category. In all of the results from this study, $\mathbf{I}_{\mathrm{s}}$ is excluded for explosion sources because the radiation pattern only produces P-waves and therefore does not allow any localisation with $\mathbf{I}_{\mathrm{s}}$. Similarly, $\mathbf{I}_{\mathrm{p}}$ is excluded for strike-slip sources because it was not possible to locate strike-slip sources with $\mathbf{I}_{\mathrm{p}}$ using a homogeneous model. Three source depths were tested with an explosion and a strike-slip source. Inter-station distances producing locations in category I or II for at least two imaging conditions were considered reliable. Category III locations may introduce ambiguities.

Inter-station distances and apertures producing reliable locations can be seen in Fig. 4. We were able to locate a strikeslip source at a depth of $5 \mathrm{~km}$ with nine receivers placed 1 to $9 \mathrm{~km}$ apart. The explosion source at a depth of $5 \mathrm{~km}$ was reliably located with an inter-station distance of $6 \mathrm{~km}$. For a $11.9 \mathrm{~km}$ deep source, a reliable localisation was possible with inter-station distances of 4 to $19 \mathrm{~km}$ for a strike-slip source and 9 to $15 \mathrm{~km}$ for an explosion source. The strike-slip source at a depth of $22 \mathrm{~km}$ was located with nine receivers spaced 17 to $25 \mathrm{~km}$ apart and the explosion source with receivers spaced 15 to $25 \mathrm{~km}$ apart. The strike-slip source at a depth of

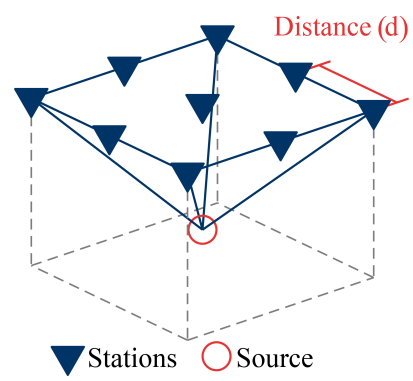

Figure 3. Placement of the nine stations used as receivers for testing the influence of the inter-station distance $(d)$. The middle receiver is directly above the source.

$22 \mathrm{~km}$ could also be located with inter-station distances of 6 to $12 \mathrm{~km}$. However, at an inter-station distance of $15 \mathrm{~km}$ there was only one imaging condition that led to a reliable localisation. Therefore only the larger inter-station distances were considered successful for this source depth.

In general, we found that sources at a certain depth could be located with an inter-station distance roughly the same as the source depth and an aperture of twice the source depth which relates to an aperture angle of 90 to $100^{\circ}$. Additionally, the range for optimal apertures decreased with increasing source depths for strike slip sources but increased with increasing source depths for explosion sources. Furthermore, strike-slip sources could be located with a wider range of inter-station distances than explosion sources.

In our set-up with a homogeneous velocity model, nine receivers were sufficient to reliably locate explosion and strikeslip sources. In Fig. 5, the localisation results for an increased number of receivers are shown. Receivers were added outside of the nine original receivers at the same inter-station distance. Receiver distances remained constant $(13 \mathrm{~km}$ for this source at a depth of $11.9 \mathrm{~km}$ ) while the aperture was increased. Additionally, receivers were added inside the original array; consequently, the aperture was kept constant at $24 \mathrm{~km}$

Increasing the number of receivers from 9 to 25 increased the accuracy of the localisation. Forty-nine receivers slightly enhanced the results further. Using 169 receivers, spaced $2 \mathrm{~km}$ apart, did not further improve the location accuracy. For the strike-slip source the location accuracy even decreased to a category III location for $\mathbf{I}_{\mathrm{e}}$. These results suggest that a slight improvement in location accuracy can be achieved using more stations. Nevertheless, nine stations produced reliable locations with a homogeneous velocity model and no noise in the traces. The only simulations in this study where all imaging conditions produced a category I location can be seen in Fig. 5 for the strike-slip source when using 25 or 49 stations. An explosion source could not be located with three category I locations.

In addition to the optimal range of inter-station distances, we found that different imaging conditions were sensitive to 


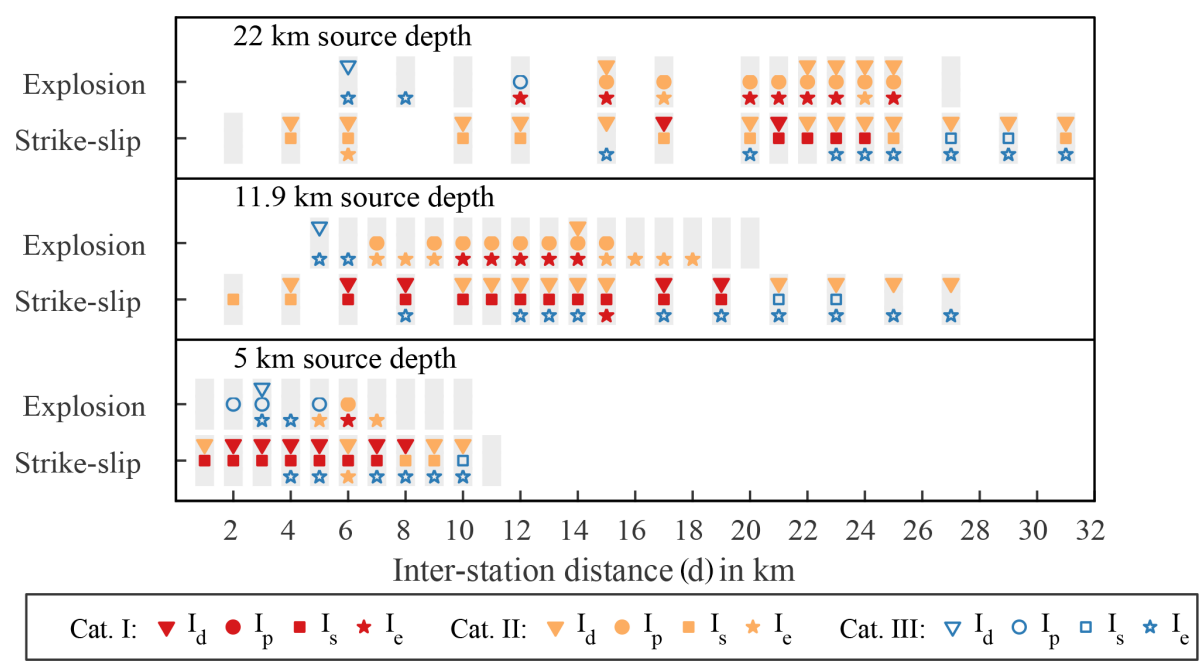

Figure 4. The location accuracy achieved with different inter-station distances $(d)$. Three source depths were tested for the two source types, respectively. The location accuracies achieved with the individual imaging conditions were ranked according to the categories from Fig. 2. Grey bars indicate set-ups that were tested. No symbol in the grey bar means that the location was unsuccessful. Symbol types and colours in the grey bars represent successful locations with the imaging condition sorted into the respective category.
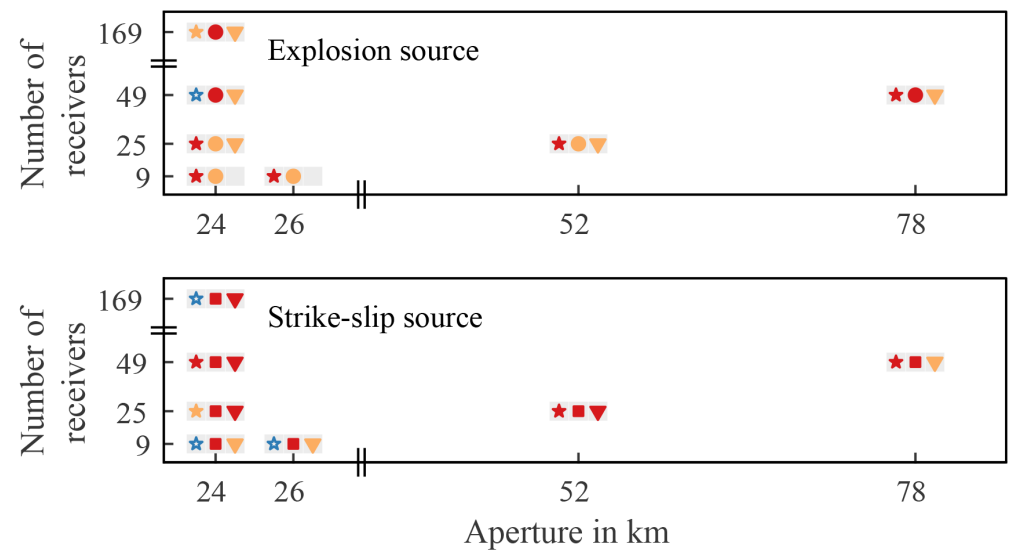

Cat. I: $\nabla \mathrm{I}_{\mathrm{d}} \bullet \mathrm{I}_{\mathrm{p}} \bullet \mathrm{I}_{\mathrm{s}} \star \mathrm{I}_{\mathrm{e}} \quad$ Cat. II: $\nabla \mathrm{I}_{\mathrm{d}} \bullet \mathrm{I}_{\mathrm{p}} \backsim \mathrm{I}_{\mathrm{s}} \star \mathrm{I}_{\mathrm{e}} \quad$ Cat. III: $\nabla \mathrm{I}_{\mathrm{d}} \circ \mathrm{I}_{\mathrm{p}} \square \mathrm{I}_{\mathrm{s}} \star \mathrm{I}_{\mathrm{e}}$

Figure 5. The location accuracy achieved with an increased number of stations. Two source types were tested at a depth of $11.9 \mathrm{~km}$. Receivers were either added between the nine existing stations (aperture stays the same) or outside of the nine existing receivers (aperture increases). The locations achieved with the individual imaging conditions were ranked according to the categories from Fig. 2. Grey bars indicate set-ups that were tested. No symbol in the grey bar means that the location was unsuccessful. Symbol types and colours in the grey bars represent successful locations with the imaging condition sorted into the respective category.

different source types. While $\mathbf{I}_{\mathrm{d}}$ and $\mathbf{I}_{\mathrm{s}}$ seemed to be successful for a strike-slip source, $\mathbf{I}_{\mathrm{e}}$ and $\mathbf{I}_{\mathrm{p}}$ were more successful for an explosion source. This can be seen particularly well when considering only the category I locations in Figs. 4 and 5.

\subsection{Arrays not centred above the source location}

To determine the sensitivity of TRI to an event not centred beneath the array, locations were analysed with an array of nine receivers shifted in one direction. In practice, arrays are rarely centred above an event location. Therefore, it is neces- sary to know where in the model sources can be located. This helps to estimate where to put stations if events are expected in a specific target area or where locations can be successful when using a preexisting array.

In this section, the asymmetry of the array in relation to the source location was investigated. Nine receivers were placed in a square using the inter-station distances that produced reliable locations in the previous section. In Table 1, the inter-station distance used for each source depth can be seen. These nine receivers were moved in negative $x$ direction in order to increase the shift distance $(s)$ between the centre re- 
Table 1. Receiver distances used for different source depths according to results from Sect. 3.1.

\begin{tabular}{rr}
\hline Source depth $(\mathrm{km})$ & Receiver distance $d(\mathrm{~km})$ \\
\hline 5 & 6 \\
11.9 & 13 \\
22 & 23 \\
\hline
\end{tabular}

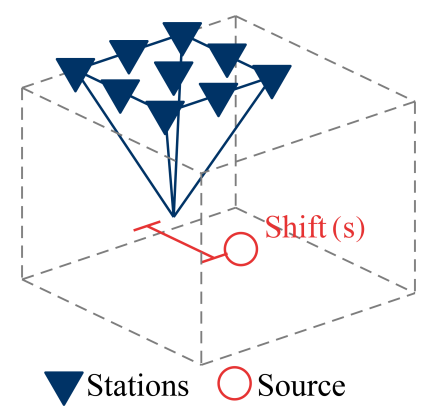

Figure 6. The placement of the nine stations used as receivers for testing the influence of an array which is not centred above the source. The shift $(s)$ was discretely increased.

ceiver and the source location (see Fig. 6). For smaller shift distances some part of the array is still above the source. At one shift $s$ the shift distance is equal to the inter-station distance. At this point one of the side receivers is directly above the source location. For larger shifts the source location is beside the array. In Fig. 7 the shift distance $(s)$ where the source is no longer beneath the array is marked by a dashed line.

In Fig. 7 the results for different shift distances $(s)$ are shown. A $0 \mathrm{~km}$ shift represents an array that is centred above the source location. Shallow explosion sources, at a depth of $5 \mathrm{~km}$, could not be located with an array not centred above the source while strike-slip sources at a depth of $5 \mathrm{~km}$ could be located with a shift $s$ of $6 \mathrm{~km}$. Reliable locations were possible for explosion sources at a depth of $11.9 \mathrm{~km}$ at shifts of 6 and $8 \mathrm{~km}$, whilst strike-slip sources at a depth of $11.9 \mathrm{~km}$ could be located with shifts of 2,13 and $19 \mathrm{~km}$. Between these shift distances strike-slip sources could either only be located with one imaging condition or not at all. Explosion sources at a depth of $22 \mathrm{~km}$ could be located with shifts from 8 to $13 \mathrm{~km}$, while strike-slip sources at this depth could be located with shifts of 20 and $23 \mathrm{~km}$.

Explosion sources could be most successfully located when the centre of the array was directly above the source or when the source was between the receivers; it could not be reliably located if the source location was too close to one of the receivers or beside the array. Strike-slip sources could be located if one of the receivers of the array was above or near the source location, but they could not be located if the source location was between two stations. Neither the explosion source nor the strike-slip source could be located when the source location was outside of the array. The only excep- tion was the strike-slip source at a depth of $11.9 \mathrm{~km}$ : it could be reliably located with a shift of $19 \mathrm{~km}$.

To further investigate the different behaviour of the localisation of explosion and strike-slip sources, simulations were performed with an increased number of receivers. Twentyfive receivers were placed in a $5 \times 5$ square. The inter-station distance was set to $8 \mathrm{~km}$ which is smaller than the interstation distance used for the nine receivers in Fig. 7. An explosion and a strike-slip source at a depth of $11.9 \mathrm{~km}$ were tested. The results can be seen in Fig. 8. For both source types almost all tested shifts of the array were able to locate the sources reliably. There was no significant difference between simulations where one of the receivers was above the source location $(s=8 \mathrm{~km}$ and $s=16 \mathrm{~km})$ and those where the source location was between the receivers $(s=4 \mathrm{~km}$ and $s=12 \mathrm{~km}$ ). From a shift distance of $16 \mathrm{~km}$ onwards, the source was beside the array. Both source types could be reliably located when the source was next to the array up to a shift of $32 \mathrm{~km}$ where the source was $16 \mathrm{~km}$ from the array. At a shift of $24 \mathrm{~km}$, the explosion source could no longer be reliably located, while at a shift of $28 \mathrm{~km}$ the strike-slip source could no longer be reliably located. An overall decrease in location accuracy was observed when the source was not beneath the array.

Overall, an increased number of receivers with a smaller inter-station distance ensured the localisation of strike-slip and explosion sources beneath the whole array. Additionally, a larger array also enabled the localisation of events outside of the array. Conclusively, if the source locations are not known a rather large array should be used.

\subsection{Irregular station distributions}

To determine the influence of heterogeneous inter-station distances on the localisation capabilities of TRI, the azimuthal gap $(a)$ was used. Seismic stations are rarely placed in a square with every receiver the same distance apart. Horstmann et al. (2015) introduced the azimuthal gap as the horizontal angle between two stations viewed from the projection of the source to the surface. With nine receivers placed in a square, the azimuthal gap is $45^{\circ}$ for each station pair, excluding the centre station. Therefore, when moving the corner stations closer to the source location in the $x$ direction, the angle increases between some of the stations and decreases between other stations (see Fig. 9). In this study, the azimuthal gap refers to the maximum angle between stations. The minimum azimuthal gap can be derived by subtracting the maximum azimuthal gap from $90^{\circ}$ because the corner stations are moved inward from their corner positions in the $x$ direction until they meet the next station. In the end, the array is effectively reduced to five stations with homogeneous azimuthal gaps of $90^{\circ}$. In this synthetic test the array is still symmetric to the source location. However, for larger and potentially more irregular arrays the azimuthal gap indicates if there is a large gap in the array where no signal is 


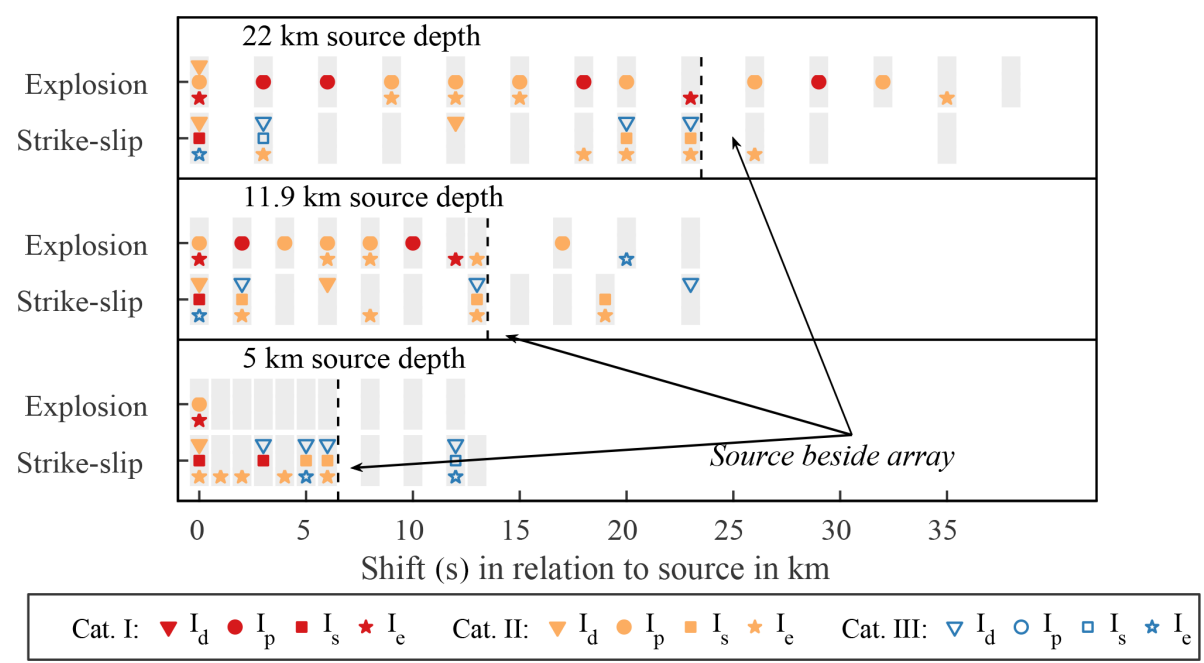

Figure 7. The location accuracy obtained with different shift distances $(s)$ between the middle of the array and the source location. The dotted line denotes the point at which the array is no longer above the source. Three source depths were tested for the two source types, respectively. The locations achieved with the individual imaging conditions were ranked according to the categories from Fig. 2. Grey bars indicate set-ups that were tested. No symbol in the grey bar means that the localisation was unsuccessful. Symbol types and colours in the grey bars represent successful locations with the imaging condition sorted into the respective category.

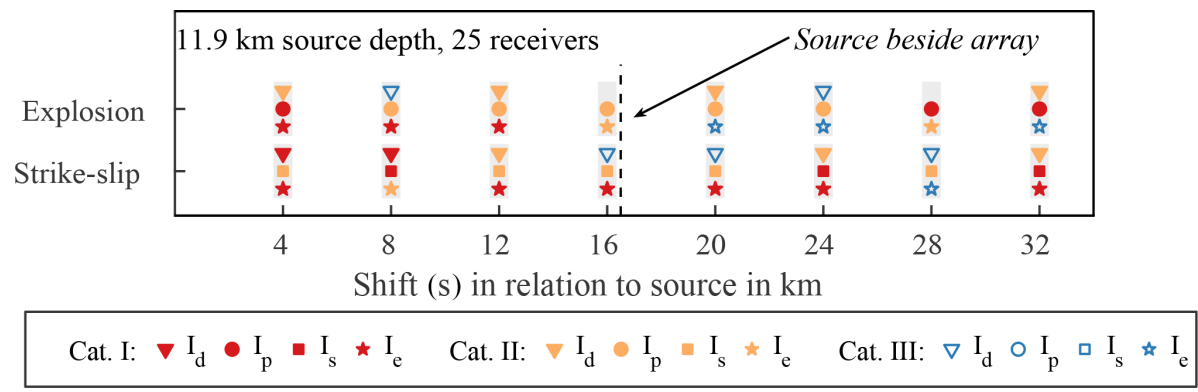

Figure 8. The location accuracy achieved using 25 stations instead of 9 stations placed $8 \mathrm{~km}$ apart while shifting the array. The dotted line denotes the point at which the array is no longer above the source. A source at a depth of $11.9 \mathrm{~km}$ was tested with two source types. The locations obtained with the individual imaging conditions were ranked according to the categories from Fig. 2. Grey bars indicate set-ups that were tested. No symbol in the grey bar means the localisation was unsuccessful. Symbol types and colours in the grey bars represent successful locations with the imaging condition sorted into the respective category.

recorded. Depending on the size of this gap, there may not be enough recorded waveforms from that direction; therefore, the source reconstruction may become impossible.

The localisation results are shown in Fig. 10. As the stations could only be moved on the model grid, the resulting azimuthal gaps are spaced differently for the three source depths. We found that the deeper the source the larger the azimuthal gap. For a $22 \mathrm{~km}$ deep source, reliable locations were possible with a maximum azimuthal gap of up to $67^{\circ}$. An $11.9 \mathrm{~km}$ deep source could be reliably located with an azimuthal gap of up to $58^{\circ}$. The shallow source, at a depth of $5 \mathrm{~km}$, did not seem to allow a heterogeneous station distribution.

\section{The effect of a complex velocity model}

In addition to the station distribution, a complex velocity model may have a strong influence on the localisation capabilities of TRI. In general, a sufficiently accurate velocity model is needed to be able to adequately back propagate the wave field. However, a sufficiently correct velocity model may also include complex velocity structures such as low velocity zones or sharp velocity contrasts. These structures can trap energy and obscure the source location. This is why the imaging conditions were divided by an illumination map as described in Sect. 2. Nevertheless, pronounced artefacts may remain in the final result.

To investigate the extent of focusing errors due to complex velocity structures, four different velocity models were tested. Differently shaped and positioned low velocity zones 


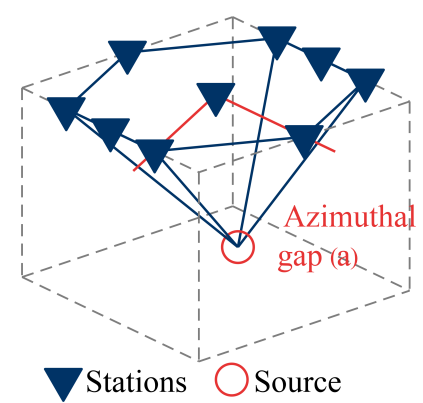

Figure 9. Placement of the nine stations used as receivers for testing the influence of stations that are heterogeneously distributed. The maximum azimuthal gap $(a)$ is the largest angle found between two stations viewed from the centre station. The azimuthal gap was increased by moving the corner stations closer to the side receivers on two sides.

were tested as well as a fault. Each velocity model was tested once with the low velocity zone or fault known and then again with an unknown low velocity zone or fault. The velocities in the low velocity zones were $75 \%$ of the values from the homogeneous models $\left(v_{\mathrm{p}}=4000 \mathrm{~m} \mathrm{~s}^{-1}\right)$. The layered model used four layers and $\mathrm{P}$-wave velocities ranged from 3000 to $6000 \mathrm{~km}$. S-wave velocities were calculated with a constant ratio of $v_{\mathrm{s}}=v_{\mathrm{p}} / \sqrt{3}$.

The forward simulation incorporated the structure, while one backward propagation was undertaken with the exact same velocity model as the forward simulation and one was carried out with a homogeneous or layered velocity model instead. For each velocity model, a strike-slip source and an explosion source were tested at a depth of $11.9 \mathrm{~km}$. Two sets of receivers, one with 9 receivers and one with 25 receivers, were placed in a square and centred above the source. The receivers were spaced $13 \mathrm{~km}$ apart for both sets.

The localisation results are shown in Fig. 11. The results of the two sets of receivers used are shown in the upper and middle panel. The velocity model used for the forward simulation and one of the time-reversed simulations is shown in the lower panel. For the second time-reversed simulation a homogeneous model or a simple layered model was used.

When there was a known low velocity layer above the source (see Fig. 11, left model), both a strike-slip source and an explosion source could be reliably located. There is no drastic improvement when using more receivers. When the low velocity layer above the source was unknown, the localisation capabilities decreased and a reliable location was no longer possible.

When the source was inside the low velocity layer (see Fig. 11, second model), the localisation was not significantly enhanced by using more receivers. When this low velocity zone was unknown, the reliable localisation of a strike-slip source was still possible but the localisation of an explosion source was no longer possible.
When there was a spherically shaped low velocity zone around the source (see Fig. 11, second model from the right), the localisation was enhanced when using 25 receivers. A strike-slip source inside a spherically shaped low velocity zone could be located when the low velocity zone was known but not when it was unknown; however, an explosion source could still be located when the low velocity zone was unknown.

The last velocity model was a layered model with a fault (see Fig. 11, right model). When the fault was known the strike-slip source could be located with 9 and 25 receivers. When it was unknown, and just a layered velocity model was used for the backward propagation, the reliability decreased and the source could no longer be located. An explosion source could not be located successfully, even with 25 receivers.

Taken together these results suggest that an increased number of receivers only helped slightly when the velocity model was more complex. Additionally, the localisation of strike-slip sources was influenced less by a complex velocity model than the localisation of explosion sources. If structures like low velocity zones and faults were known, it did not hinder the localisation; however, if they were unknown, location accuracy decreased.

\section{Limits for the signal-to-noise ratio}

The previous sections only used synthetic seismograms recorded during forward simulations and then reinserted into the model time-reversed. However, real data does not only consist of the signal corresponding to the event. It also includes a variable amount of noise. In theory, TRI works with noisy data (Gajewski and Tessmer, 2005; Witten and Artman, 2011). In this section, the smallest signal-to-noise ratio (SNR) still enabling source locations was investigated. An explosion source and a strike-slip source at a depth of $11.9 \mathrm{~km}$ were used. Nine and twenty-five receivers were spaced $13 \mathrm{~km}$ apart and centred above the source. Five different SNR values were used: $1,0.75,0.5,0.25$ and 0.1 .

The noisy data was created by constructing a time series with random amplitudes and the same time step and length as the seismograms. Next the noise signal was filtered to exclude numerically unstable frequencies above $5 \mathrm{~Hz}$. The amplitudes of this time series were modified to achieve the desired SNR of this noise signal in relation to the seismograms of all stations. The filtered noise signal was then added to the seismograms of all stations. Figure 12 shows example traces of the seismograms without noise and the seismograms with the respective SNR value for a receiver directly above an explosion source. As the SNR decreased, the event signal became less and less distinguishable from the noise.

The localisation results are shown in Figure 13. When using nine receivers there was no reliable location possible for both source types. With a SNR of 1 there was only one loca- 


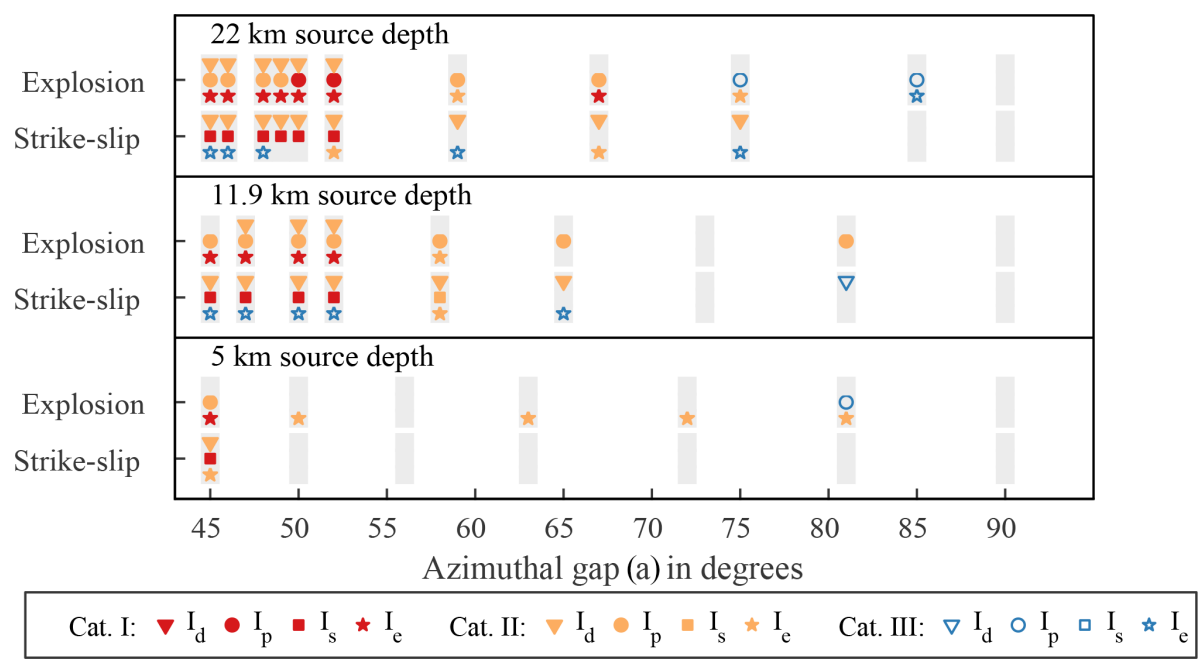

Figure 10. The location accuracy achieved with different maximum azimuthal gaps $(a)$. Three source depths were tested for the two source types, respectively. The locations obtained with the individual imaging conditions were ranked according to the categories from Fig. 2. Grey bars indicate set-ups that were tested. No symbol in the grey bar means that the localisation was unsuccessful. Symbol types and colours in the grey bars represent successful locations with the imaging condition sorted into the respective category.

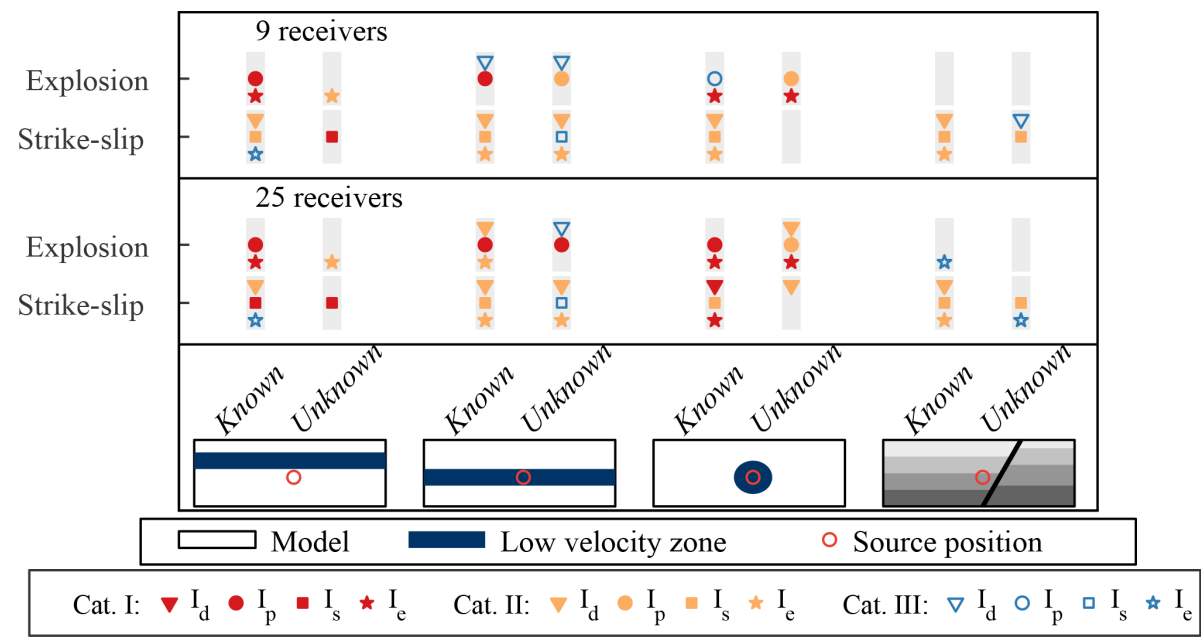

Figure 11. The location accuracy achieved with different velocity models. For each velocity model one simulation was performed with the low velocity zone or fault known, and one simulation was performed with a homogeneous or layered velocity model for the backward simulation. A set of 9 receivers and a set of 25 receivers was tested. The localisation obtained with the individual imaging conditions were ranked according to the categories from Fig. 2. Grey bars indicate set-ups that were tested. No symbol in the grey bar means the localisation was unsuccessful. Symbol types and colours in the grey bars represent successful locations with the imaging condition sorted into the respective category.

tion in category II, whilst for SNRs down to 0.5 there were only locations in category III. When using 25 receivers it was possible to locate both source types with a SNR as low as 0.25 in category I but only with one imaging condition. Explosion sources could be located in category I with two different imaging conditions with a SNR as low as 0.5. Surprisingly, the total energy density imaging condition $\mathbf{I}_{\mathrm{e}}$ seemed to be the most stable for locating sources of both source types. In the previous sections, $\mathbf{I}_{\mathrm{e}}$ did not seem to be as suitable for a strike-slip source as the other imaging conditions.
Overall, these results suggest that the noise level has a higher impact on location accuracy than complex velocity models. It was possible to locate sources even if the noise level was too high to distinguish events in the traces. Nevertheless very low SNRs hinder a reliable localisation which can only partly be compensated for by adding more receivers. 


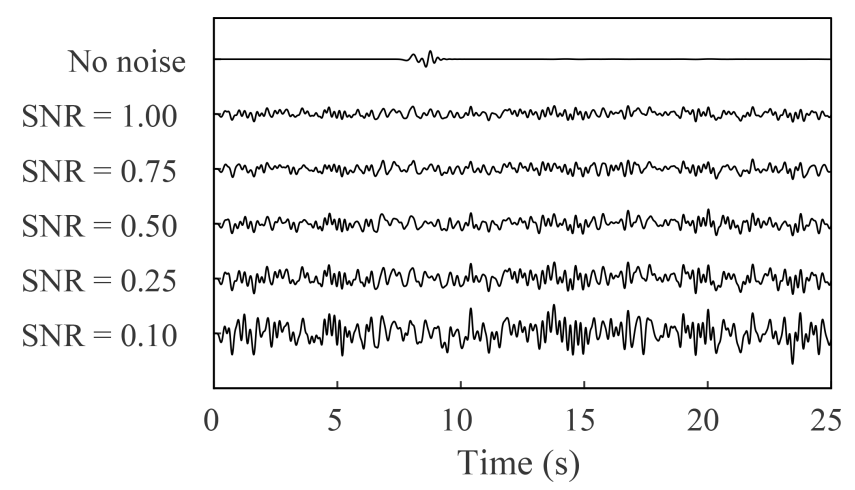

Figure 12. Example traces recorded with a receiver directly above the source for an explosion source placed at a depth of $11.9 \mathrm{~km}$. The traces without noise and with different degrees of noise are shown, resulting in different signal-to-noise ratios (SNR).

\section{Application example for southern California}

In previous sections, station distributions were systematically tested to gain insight into the characteristics of array designs that influence localisation results. Additionally, results showed that noisy data influence the localisation more than a complex velocity model if the velocity model is accurate enough. In this section, a real-life scenario from southern California is mimicked by simulating synthetic seismograms with a real velocity model and real station positions. The advantage of using synthetic data is that the true source location is known; therefore, the location accuracy can be seen directly. First, the given stations are evaluated for their ability to locate events with a homogeneous velocity model. We show that subsets of the array may enhance the location accuracy; we also show how to design an array in this region. Second, we estimate the success rate achievable using different station distributions, the velocity model by Zeng et al. (2016) and noisy data.

\subsection{Determine localisation possibilities using results from previous sections}

Horstmann et al. (2015) located non-volcanic tremors in southern California near Cholame using 39 stations. In this study, we utilised 38 of the 39 stations (the northernmost station was excluded here) and tried to locate synthetic events. The receiver positions were transformed to the same $x-y$ grid as in Horstmann et al. (2015) and plotted in Fig. 14a. Additionally, three source positions were plotted. These source positions are used in the following to test the possibility of locating events with one of three receiver sets derived from the 38 stations in southern California and two receiver sets suggested as optimal arrays following the results of the previous sections. Strike-slip sources were used as the source type in this section. Strike-slip sources occur more dominantly in the subsurface than explosion sources. The extent of this model in $x$ and $y$ directions is the same as in previous models as well as in Horstmann et al. (2015).

Figure 14a shows that the 38 irregular stations extend further in the $y$ direction than in the $x$ direction. As the receivers are so heterogeneously spaced, this set of receivers was not expected to give a reliable localisation for any of the three sources. However, the total number of stations may counteract the heterogeneous inter-station distances. Additionally, we determined two subsets of receivers with more homogeneous inter-station distances. Two subsets were chosen here: one with 31 stations (see Fig. 14b) and one with 20 stations (see Fig. 14c). For receiver set (b) stations very close to each other were excluded, while for set (c) stations were reduced to decrease the total amount of traces, which may impact computation time. Lastly, in Fig. 14d and e two optimal arrays are suggested, one with 32 stations and one with 20 stations. These arrays were designed with a homogeneous station distance of $8 \mathrm{~km}$. The arrays were positioned to allow locations in roughly the same areas as in Fig. 14b and c. For a real-life applications, the arrays would be moved to locate events in the specific target area. The inter-station distances and apertures for the arrays can be found in Table 2.

The target depth for locations was between 10 and $25 \mathrm{~km}$ because bigger events occur at depths as shallow as $10 \mathrm{~km}$ while non-volcanic tremor signals seem to occur deeper (Horstmann et al., 2015). For this target depth range, the results from Sect. 3.1 suggested that the aperture should be about $20 \mathrm{~km}$ or larger to be able to locate deeper events, whereas the receiver distance should not be larger than about $13 \mathrm{~km}$ to include shallower events. When comparing these requirements to the values reported in Table 2, the minimum aperture was greater than $20 \mathrm{~km}$ for all four receiver sets. Similarly, the average inter-station distance was less than $13 \mathrm{~km}$ for all four receiver sets. However, the inter-station distances vary greatly, especially for receiver set (a) and (b).

In Fig. 14 the two grey circles mark areas where events are expected to be located with more or less imaging conditions in better categories. These circles were derived from the results from previous sections where we discovered that sources could be located beneath the array when using fewer stations and slightly outside the array when using an increased number of receivers. Three source positions were chosen to test where locations should be expected.

Simulations were performed with a strike-slip source and a homogeneous velocity model (see top of Fig. 15). The source was placed in all three source positions and at two depths. The five receiver sets presented in Fig. 14 were tested for their capability to locate these sources.

Locations were possible with receiver set (a), although inter-station distances were very heterogeneous. At source position 1, a reliable location was possible in a depth of $11.9 \mathrm{~km}$; at a depth of $22 \mathrm{~km}$, no reliable location could be found. At source position 2 and 3 a location at a depth of $22 \mathrm{~km}$ was possible. 


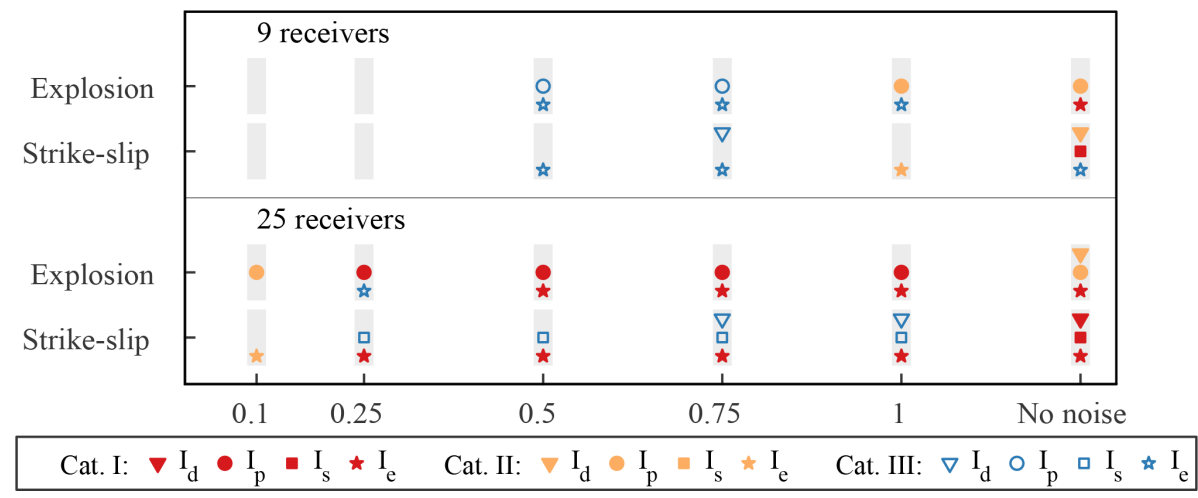

Figure 13. The location accuracy obtained with different amounts of noise added to the traces. A set of 9 receivers and a set of 25 receivers was tested for two different source types. The locations obtained with the individual imaging conditions were ranked according to the categories from Fig. 2. Grey bars indicate set-ups that were tested. No symbol in the grey bar means the localisation was unsuccessful. Symbol types and colours in the grey bars represent successful locations with the imaging condition sorted into the respective category.

(a) 38 actual receivers

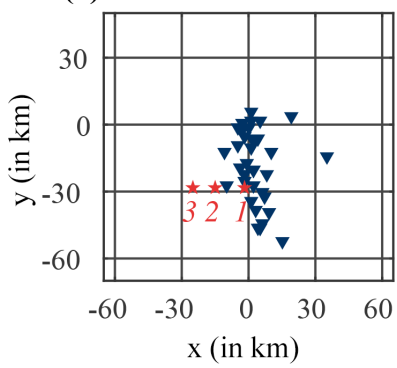

(d) 32 suggested receivers $(e)$

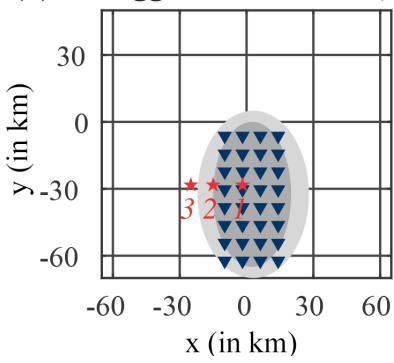

(b) 31 actual receivers

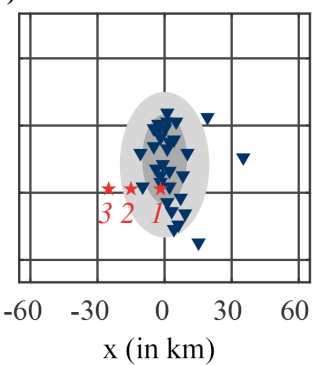

20 suggested receivers

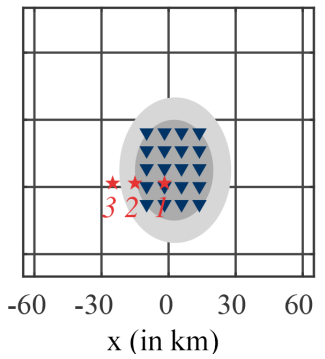

(c) 20 actual receivers

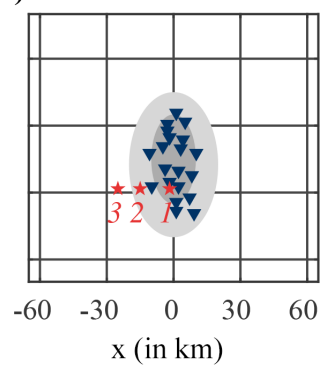

$\star$ Sources

$\nabla$ Stations

Figure 14. Receiver sets used to test the success rate of locations in southern California: (a) 38 stations as used by Horstmann et al. (2015), (b) a subset of (a) excluding stations that are very close to another station, (c) 20 stations from (a), (d) 32 suggested stations with regular inter-station distances and (e) 20 stations suggested for optimal results. Three source positions were chosen to test the range of the localisation.

Receiver set (b) has a slightly enhanced location accuracy compared to set (a). All sources could be reliably located. This highlights that TRI is rather stable, and that small alterations to the station distribution allow a localisation of events at multiple depths at different positions in the model.

Receiver set (c) consisted of 20 stations and location accuracy was decreased for the sources at a depth of $22 \mathrm{~km}$. An explanation for the decreased quality for deeper sources could be the reduced aperture of this receiver set. However, the source at a depth of $11.9 \mathrm{~km}$ could be reliably located with all three imaging conditions in category I and II. This is in agreement with previous results suggesting a decrease in the sensitivity to station distribution with depth.

Receiver sets (d) and (e) were suggested as optimal arrays. In comparison to receiver set (b), receiver set (d) produced a higher location accuracy while receiver set (e) resulted in slightly worse locations than the irregularly distributed stations of set (c). For this homogeneous case, the suggested array with 32 stations and regular inter-station distances produced the most accurate locations.

After performing this synthetic study with a homogeneous velocity model, we would recommend the use of receiver set (b) for locating events in this region if the stations used in 
Table 2. Inter-station distances $(d)$ and apertures $(a)$ of the five receiver sets shown in Fig. 14.

\begin{tabular}{lrrrrrr}
\hline Receiver set (from Fig. 14) & Number of stations & Min $d(\mathrm{~km})$ & Max $d(\mathrm{~km})$ & Mean $d(\mathrm{~km})$ & Min $a(\mathrm{~km})$ & Max $a(\mathrm{~km})$ \\
\hline (a) & 38 & 0.138 & 23.94 & 3.919 & 45.369 & 58.487 \\
(b) & 31 & 2.002 & 23.94 & 5.224 & 45.369 & 58.487 \\
(c) & 20 & 2.901 & 7.499 & 5.024 & 20.348 & 44.923 \\
(d) & 31 & 8 & 8 & 8 & 24 & 56 \\
(e) & 20 & 8 & 8 & 8 & 24 & 32 \\
\hline
\end{tabular}

\begin{tabular}{|c|c|c|c|c|c|c|}
\hline Source 1 & $11.9 \mathrm{~km}$ depth & $* \nabla$ & $\star \square \nabla$ & $\star \pm \nabla$ & $* \pi$ & $\star \square \nabla$ \\
\hline $\begin{array}{l}\text { Source } 1 \\
\text { Source } 2 \\
\text { Source } 3\end{array}$ & $22 \mathrm{~km}$ depth & 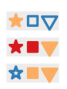 & 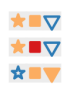 & $\begin{array}{l}\frac{\hbar \square}{\star \square \square} \\
\star \square \nabla V\end{array}$ & $\begin{array}{l}\star \square \nabla \\
\star \square \nabla \\
\star \square\end{array}$ & $\begin{array}{l}\text { समV } \\
\text { सवV }\end{array}$ \\
\hline
\end{tabular}

$\begin{array}{lllll}\text { Receiver set: } & \text { (a) } & \text { (b) } & \text { (c) } & \text { (d) }\end{array}$

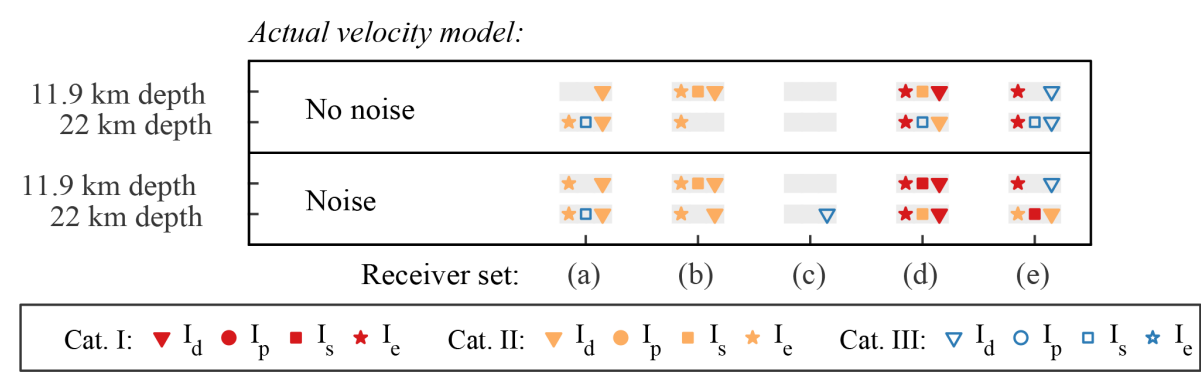

Figure 15. The location accuracy achieved with a strike-slip source at different depths, with the different receiver sets and a homogeneous velocity model (top half) and with the velocity model of Zeng et al. (2016) and added noise $(\mathrm{SNR}=1)$ (bottom half of the figure). The lower case letters represent the receiver sets of Fig. 14. The localisation obtained with the individual imaging conditions were ranked according to the categories from Fig. 2. Grey bars indicate set-ups that were tested. No symbol in the grey bar means the localisation was unsuccessful. Symbol types and colours in the grey bars represent successful locations with the imaging condition sorted into the respective category.

this study are the only available stations. If stations were to be deployed, receiver set (d) would be recommended.

\subsection{Synthetic tests to estimate the success rate with real data}

After finding a suitable receiver set that enabled localisation with a homogeneous velocity model at different source depths and different source positions, the success rate with the real velocity model and noisy data needed to be investigated. Therefore, additional simulations were performed.

Zeng et al. (2016) present the most current and accurate velocity model for the chosen region in southern California in which Horstmann et al. (2015) locate non-volcanic tremors. The velocity model is three-dimensional and incorporates Pand $\mathrm{S}$-wave velocities. It was interpolated to fit the finite difference grid utilised, and the density was calculated from the $\mathrm{P}$-wave velocity with the empirical equation from Brocher (2005):

$$
\begin{aligned}
& \rho=1.6612 v_{\mathrm{p}}-0.4721 v_{\mathrm{p}}^{2}+0.0671 v_{\mathrm{p}}^{3} \\
& -0.0043 v_{\mathrm{p}}^{4}+0.000106 v_{\mathrm{p}}^{5},
\end{aligned}
$$

where $v_{\mathrm{p}}$ depicts the $\mathrm{P}$-wave velocity in $\mathrm{kms}^{-1}$ and the resulting density $\rho$ is in $\mathrm{g} \mathrm{cm}^{-3}$.

At the bottom of Fig. 15, localisation results for simulations with and without added noise are shown. Without any noise, the strike-slip source at position number 1 could be reliably located at a depth of $11.9 \mathrm{~km}$ with receiver set (b) and at a depth of $22 \mathrm{~km}$ with receiver set (a). Receiver set (c) was not able to produce any location with the velocity model. The suggested receiver sets (d) and (e) also produced more accurate locations in these cases with the real velocity model and no noise.

When adding noise with a SNR of 1 , reliable localisations were possible at both depths with receiver sets (a) and (b). Receiver set (c) was still unable to locate any of the sources; however, receiver sets (d) and (e) were able to locate the sources. Receiver set (d) even produced a category I location for all three imaging conditions.

Receiver set (b) remained the best choice in this case followed by receiver set (a). Both were able to locate three out of four sources. Both sets reliably located the sources with noise. Nevertheless, the suggested arrays with regular inter- 
station distances were more successful than the irregular arrays.

We would suggest the use of receiver set (b) to locate events in this area and avoid ambiguity, as all locations were in category II and there were no category III locations. The success rate of locations in this case is expected to be high inside the grey circles marked in Fig. 14 as long as the noise level is not too high. Location accuracy is expected to decreases with increasing distance from the array. However, if new stations were to be deployed, we would recommend placing them in a similar fashion to receiver set (d).

\section{Discussion}

The objective of this study was to investigate the sensitivity of TRI to station distributions while considering complex velocity models and a low signal-to-noise ratio. This is important for (1) estimating the success rate of TRI with a given array and velocity model, (2) decreasing the time needed to adjust the stations (e.g. by choosing only a subset of receivers) and (3) designing an array for locating events in a designated target area.

The TRI method is straight-forward and can be implemented into most numerical codes which can propagate elastic waves through a medium. In this study, we further improved the workflow of Saenger (2011) which was modified by Witten and Artman (2011). We suggested visualising all points with certain fractions of the highest value in imaging conditions and proposed a set of categories to quantify the accuracy of source locations. When using real data, division into categories may be obsolete. If the receiver distribution is sufficient and the level of noise is low enough, all focusing spots appearing in the imaging conditions should represent source locations.

Additionally, we rated the success of locations by the distance between the imaged and the true source location. The threshold deviation used was calculated as the average deviation from all three directions. Therefore, successful locations for the shallowest source could be at the surface. However, surface locations were excluded. This restricted the range for a successful location of the source at a depth of $5 \mathrm{~km}$. Consequently, it seemed that a source at a depth of $5 \mathrm{~km}$ was especially hard to locate. However, sources shallower than $10 \mathrm{~km}$ are rarely observed in the chosen region in Southern California (Horstmann et al., 2015).

All simulations in this study were set up with the same frequency range for the source wavelet, the same grid spacing and the same time step. For explosion sources this setup was numerically stable in the range of seismic velocities used. However, S-waves and surface waves experience numerical dispersion which cannot be reconstructed during the back propagation of the wave field. The numerical dispersion could not be eliminated without reducing the grid spacing and consequently increasing computation time significantly.
Therefore, we kept the chosen values. We observed no effect in the results suggesting that dispersive S-waves interfered with the localisation. The surface waves were more dispersive than the $\mathrm{S}$-waves but are not relevant for this method; consequently, they also do not interfere with the localisation. Dispersive surface waves may be another reason why we needed to exclude focusing at the surface.

In the workflow used, we muted the top $2.3 \mathrm{~km}$ in the imaging conditions to remove any focusing at the surface. This does not allow for the localisation of shallow sources, but it increases the success rate for deeper sources. Other studies restricted possible focusing to the region around the source location (e.g. Horstmann et al., 2015). However, we observed that the difference between areas where locations were reliable and areas where they were not was not a clear boundary but rather a transitional zone. Additionally, depending on the velocity model and the position of the source in relation to velocity structures, waves could be scattered and sources could be located outside of this region. In contrast to reducing the area where focusing is allowed, we propose carefully evaluating focusing spots outside of the array.

\subsection{Station distribution for a reliable localisation}

Numerous simulations were performed to test different station distributions for their ability to locate sources of different source types and at different depths. In this section the results from a homogeneous velocity model without any noise added to the synthetic seismograms are discussed.

In general, we found that the inter-station distance should not be larger than the source depth to enhance localisation with TRI. As we performed all simulations with the same frequency range for the source wavelet in the forward simulation, we were unable to judge the influence of varying wavelengths on localisation results. However, inter-station distances found to produce reliable locations in this study were larger than half the dominant wavelength $(1.2 \mathrm{~km}$ in our case), which was reported as an optimal inter-station distance by Lokmer et al. (2009). Additionally, location accuracy changed with the source depth suggesting a sensitivity of the inter-station distances to the source depth. This can be explained by the discrete sampling of the back-propagated wave field. The wavefront needs to heal before the wave field can constructively interfere and focus on a source location. Witten and Artman (2011) observed that the wavefront is healed at a depth of 1 to 1.5 times the inter-station distance; consequently, sources need to be at least as deep as the interstation distance to be locatable. This is in agreement with our results.

Additionally, we found that the total aperture of the array should be at least twice the source depth. The requirement for large apertures has also been reported in previous studies (e.g. Artman et al., 2010). However, this is the first study to present a quantification of the required aperture size. 
Most simulations produced a reliable location with a homogeneous velocity model and only nine stations. More stations increased the location accuracy slightly and helped counteract the effects of a complex velocity model and high noise. However, a significantly larger number of stations did not produce perfect locations. This is contrary to most studies that report that a large number of stations is necessary for TRI (e.g. Kremers et al., 2011). We conclude that the distribution of stations may be more influential than the total number of stations on the localisation, which has also been reported by Horstmann et al. (2015).

Furthermore, we found that a smaller number of stations only enhances locations beneath the array. When the interstation distance was reduced and the number of stations was increased, events could also be located outside the array. However, the reliability of locations decreased when sources were outside of the array. This might be crucial when designing an array, as the array should be optimally positioned above the targeted source region.

In this study we used the maximum azimuthal gap between stations as an indicator of the sensitivity of location accuracy with irregular inter-station distances and found that up to a certain rate of irregularity sources could still be located. Additionally, we observed that stations could be more irregularly placed the deeper the source location. Comparing our maximum azimuthal gaps to those reported by Horstmann et al. (2015) we found that their azimuthal gaps are roughly twice the maximum azimuthal gap observed in our study. In our case with nine stations, an increase in the maximum azimuthal gap between two stations subsequently resulted in a decrease in the minimum azimuthal gap between two other stations. The discrepancy with Horstmann et al. (2015) suggests that the minimum azimuthal gap (how close two stations are to one another) has a higher influence on the localisation results than the maximum azimuthal gap. This is also supported by the improvement in location accuracy when using 31 of the 38 stations in the southern California example. Stations close to one another were excluded to form a subset of the 38 stations. Alternatively, some studies (e.g. Larmat et al., 2009 or Montagner et al., 2012) compensate for an irregular station distribution by weighing the stations according to an area assigned to each station to improve location accuracy. However, when the aim is a fast and reliable localisation method, the gain in location accuracy has to be balanced against the extra computation time needed for weighing the stations.

We performed all simulations once with an explosion source and once with a strike-slip source and observed different location accuracies for the same station distributions. We conclude that for strike-slip sources the receiver directly above the source has a greater influence on the localisation result, whereas for explosion sources the receivers further away have a greater influence. Therefore, explosion sources are more sensitive to the positioning and number of receivers, while strike-slip sources are more sensitive to how close a receiver is to the source location. This could also explain the observation of Steiner et al. (2008) that the most accurate locations can be gained when using sources that emit strong $\mathrm{S}$-waves in a vertical direction, as is the case with some strike-slip sources. It suggests that their aperture was not wide enough or that their inter-station distance was too large for locating sources emitting mainly P-waves. Furthermore, this observation dictates that inter-station distances should be small as not to produce gaps in the array where strike-slip source cannot be located.

\subsection{Evaluation of the success rate with real data}

Preliminary synthetic studies are typically used to evaluate the TRI location accuracy achievable with a certain station distribution. Often the first simulations are performed with a homogeneous velocity model. If these simulation are successful and a station distribution yields reliable results, the real velocity model is used. If that is once again successful, the real data is used which contain varying amounts of noise. Therefore, we used complex artificial velocity models and artificial noise in addition to a real velocity model from southern California to estimate the influence on the localisation.

We showed that the velocity model can be rather complex and still does not interfere with the localisation if the prominent velocity structures are known. Additionally, we observed that noisy data can hinder the localisation. An increased number of receivers enhanced the location accuracy with noisy data. However, when adding noise to the traces, the same random noise signal is added to all stations. This could unintentionally result in a structured noise that is no longer random. Nevertheless, when this noisy signal is inserted into the model it will travel in different directions and interfere differently with other signals, thus creating a noisy wave field.

Furthermore, in some cases we observed an increase in the location accuracy when using noisy data compared with simulations without noise and when using irregularly spaced stations compared to regularly spaced stations in combination with a homogeneous velocity model. This suggests that the introduction of irregularity into the simulation helps to differentiate the source signal from any other part of the waveform and enhances the focusing. Nevertheless, when mimicking a real data problem, very regular inter-station distances produced the most reliable locations.

One additional aspect that could influence the location accuracy is the impact of an asymmetric array. An asymmetric array that extends further in one direction than the other may decrease location accuracy. We observed, that it may cause the focus to be split into multiple focusing spots and therefore introduce ambiguity. Furthermore, TRI can be used for different scales and different applications. In this study we concentrated on the field scale. However, it would be a valuable addition to this study if similar studies were performed at dif- 
ferent scales to see if the same guidelines also hold true. If the same general principles apply at smaller or larger scales, it would suggest that the observed effects are inherent to the method.

In this study, the event time was known and run-times were adjusted accordingly. When using real data, the event time is unknown and therefore a strategy must also be developed to find accurate event times. The station distribution, the velocity model and the noise also possibly have an influence on the event time. This needs to be investigated in future studies. Lastly, we observed that different imaging conditions were sensitive to different source types. This could be further investigated to potentially derive the source type from results obtained while locating the source.

\section{Conclusions}

This study aimed at investigating the prerequisites for applying TRI at the field scale. Therefore, we systematically tested different station distributions with a homogeneous velocity model and evaluated the resulting locations with respect to their reliability. We focused, thereby, on deeper sources $(>5 \mathrm{~km})$ and muted the upper part of the model to eliminate artefacts.

We found that the inter-station distance should not be larger than the source depth, and that the aperture of the array should be at least twice the source depth. Additionally, sources could be most accurately located when they were underneath the array. When using more stations, locations outside of the array became possible. In general, stations should be spaced regularly, although the deeper the source the more heterogeneously the stations can be and still achieve reliable locations.

In addition to this strong sensitivity of the source location accuracy to the station distribution, we found a strong influence of the velocity model on the localisation. However, complex velocity structures, like low velocity zones or faults, do not hinder the localisation as long as they are adequately incorporated in the model. However, a high level of noise could inhibit the source location even if the velocity model was homogeneous. An increased number of stations seemed to increase location accuracy in the presence of noise.

Furthermore, we observed an increase in location accuracy in some cases with an increase in complexity, such as adding noise, more irregular stations or a more complex velocity model. This suggests that the additional scattering of waves may help the localisation. Therefore, we advise performing preliminary synthetic studies not only with homogeneous velocity models and regular stations but also incorporating as many complexities as possible to get an accurate estimation of the achievable source location.

We showed that only a few simulations are necessary when performing a preliminary synthetic study to estimate if the given set-up will work for TRI. Additionally, we showed that when designing an array to be used with TRI the target area should be considered. The depth of expected events then dictates the inter-station distance and the aperture of the array. If considering a range of depths, the inter-station distance should fit the shallowest events and the aperture the deepest events.

Data availability. The data can be accessed upon request from any of the authors.

Author contributions. CW was the lead author and wrote the paper, performed the numerical experiments and analysed the results. EHS provided the finite difference code, gave advice and support on the scope of the project, provided background knowledge and helped with editing the paper.

Competing interests. The authors declare that they have no competing interests.

Acknowledgements. This project received funding from the European Union's Horizon 2020 research and innovation programme under grant agreement no. 727550 (http://www.gemex-h2020.eu, last access: 12 December 2018) and the "Landesprogramm für Geschlechtergerechte Hochschule des Landes NRW" from the Ministry of Innovation, Science and Research of North RhineWestphalia, Germany. The authors gratefully acknowledge the computing time granted by the John von Neumann Institute for Computing (NIC) and provided on the JURECA supercomputer at the Jülich Supercomputing Centre (JSC), Germany. Furthermore, the authors would like to thank the two anonymous reviewers for their invaluable help in improving this paper.

Edited by: Michal Malinowski

Reviewed by: two anonymous referees

\section{References}

Anderson, B. E., Griffa, M., Ulrich, T. J., and Johnson, P. A.: Time reversal reconstruction of finite sized sources in elastic media, J. Acoust. Soc. Am., 130, EL219-EL225, https://doi.org/10.1121/1.3635378, 2011.

Artman, B., Podladtchikov, I., and Witten, B.: Source location using time-reverse imaging, Geophys. Prospect., 58, 861-873, https://doi.org/10.1111/j.1365-2478.2010.00911.x, 2010.

Brocher, T. M.: Empirical relations between elastic wavespeeds and density in the Earth's crust, B. Seismol. Soc. Am., 95, 20812092, https://doi.org/10.1785/0120050077, 2005.

Clayton, R. W. and Engquist, B.: Absorbing boundary conditions for wave-equation migration, Geophysics, 45, 895-904, https://doi.org/10.1190/1.1441094, 1980.

Dougherty, M. E. and Stephen, R. A.: Seismic energy partitioning and scattering in laterally heterogeneous ocean crust, Pure Appl. 
Geophys., 128, 195-229, https://doi.org/10.1007/BF01772597, 1988.

Fink, M., Cassereau, D., Derode, A., Prada, C., Roux, P., Tanter, M., Thomas, J.-L., and Wu, F.: Time-reversed acoustics, Reports Prog. Phys., 281, 91-97, 1999.

Gajewski, D. and Tessmer, E.: Reverse modelling for seismic event characterization, Geophys. J. Int., 163, 276-284, https://doi.org/10.1111/j.1365-246X.2005.02732.x, 2005.

Harker, B. M. and Anderson, B. E.: Optimization of the array mirror for time reversal techniques used in a halfspace environment, J. Acoust. Soc. Am., 133, EL351-EL357, https://doi.org/10.1121/1.4798268, 2013.

Horstmann, T., Harrington, R. M., and Cochran, E. S.: Using a modified time-reverse imaging technique to locate low-frequency earthquakes on the San Andreas Fault near Cholame, California, Geophys. J. Int., 203, 1207-1226, https://doi.org/10.1093/gji/ggv337, 2015.

Kocur, G. K., Saenger, E. H., Grosse, C. U., and Vogel, T.: Time reverse modeling of acoustic emissions in a reinforced concrete beam, Ultrasonics, 65, 96-104, https://doi.org/10.1016/j.ultras.2015.10.014, 2016.

Kremers, S., Fichtner, A., Brietzke, G. B., Igel, H., Larmat, C., Huang, L., and Käser, M.: Exploring the potentials and limitations of the time-reversal imaging of finite seismic sources, Solid Earth, 2, 95-105, https://doi.org/10.5194/se-2-95-2011, 2011.

Larmat, C. S., Montagner, J. P., Fink, M., Capdeville, Y., Tourin, A., and Clévédé, E.: Time-reversal imaging of seismic sources and application to the great Sumatra earthquake, Geophys. Res. Lett., 33, L19312, https://doi.org/10.1029/2006GL026336, 2006.

Larmat, C. S., Tromp, J., Liu, Q., and Montagner, J. P.: Time reversal location of glacial earthquakes, J. Geophys. Res.-Solid, 113, 1-9, https://doi.org/10.1029/2008JB005607, 2008.

Larmat, C. S., Guyer, R. A., and Johnson, P. A.: Tremor source location using time reversal: Selecting the appropriate imaging field, Geophys. Res. Lett., 36, 2-7, https://doi.org/10.1029/2009GL040099, 2009.
Lokmer, I., O’Brien, G. S., Stich, D., and Bean, C. J.: Time reversal imaging of synthetic volcanic tremor sources, Geophys. Res. Lett., 36, 1-6, https://doi.org/10.1029/2009GL038178, 2009.

Montagner, J. P., Larmat, C., Capdeville, Y., Fink, M., Phung, H., Romanowicz, B., Clévédé, E., and Kawakatsu, H.: Time-reversal method and cross-correlation techniques by normal mode theory: A three-point problem, Geophys. J. Int., 191, 637-652, https://doi.org/10.1111/j.1365-246X.2012.05619.x, 2012.

NCEDC: Northern California Earthquake Data Center, UC Berkeley Seismological Laboratory, Dataset, https://doi.org/10.7932/NCEDC, 2014.

Saenger, E. H.: Time reverse characterization of sources in heterogeneous media, NDT E. Int., 44, 751-759, https://doi.org/10.1016/j.ndteint.2011.07.011, 2011.

Saenger, E. H., Gold, N., and Shapiro, S. A.: Modeling the propagation of elastic waves using a modified finite-difference grid, Wave Motion, 31, 77-92, https://doi.org/10.1016/S01652125(99)00023-2, 2000.

Shearer, P. M.: Introduction to Seismology, Cambridge University Press, New York, 2nd Edn., 2009.

Steiner, B., Saenger, E. H., and Schmalholz, S. M.: Time reverse modeling of low-frequency microtremors: Application to hydrocarbon reservoir localization, Geophys. Res. Lett., 35, L03307, https://doi.org/10.1029/2007GL032097, 2008.

Witten, B. and Artman, B.: Signal-to-noise estimates of time-reverse images, Geophysics, 76, MA1-MA10, https://doi.org/10.1190/1.3543570, 2011.

Zeng, X., Thurber, C. H., Shelly, D. R., Harrington, R. M., Cochran, E. S., Bennington, N. L., Peterson, D., Guo, B., and McClement, K.: 3-D P- and S-wave velocity structure and low-frequency earthquake locations in the Parkfield, California region, Geophys. J. Int., 206, 1574-1585, https://doi.org/10.1093/gji/ggw217, 2016. 